\title{
Mortalidad infantil, Chile 1994
}

\author{
Nelson A. Vargas C. ${ }^{1}$
}

\section{Infant mortality, Chile 1994}

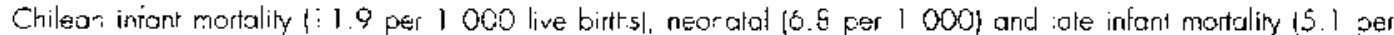
1000 rates for year 1994 , at the whole country and the 20 state-funded health systems cre presented. Counly figures reached the lowest values ever obsenved s nce reliable nohono! records are kept. :mportant variations are found among d'fierent areas of the country, building-up larger more homogeneus rates regions. To get a more accurare description of current tends, nolional rares ore presented tor the periad 1980-1994.
\end{abstract}

(Key words: infank mortality, reoriatal mortality.)

La Sociedad Chilena de Pediatría sostiene, desde hace años, un diálogo constante con numerosas instituciones del quehacer nacional, entre ellas -y como es natural- con el Ministerio de Salud, a través de diferentes comisiones y grupos técnicos. En este intercambio la sociedad aporta sus opiniones técnicas y recibe información sobre la gestión del Ministerio. Este artículo presenta, con fines de difusión y en forma sucinta, parte de la información sobre mortalidad infantil del pais que el Ministerio ha entregado en algunas reuniones de noviembre de 1995'.

Natalidad. En 1994 se observó una reducción de la tasa de natalidad, que presentó el valor más bajo registrado alguna vez en la estadística nacional (tabla I). Los nacidos vivos se redujeron en cerca de 20000 , en 1990. Datos no presentados muestran que los nacimientos siguieron concentrados en el primer y segundo orden de nacimiento $(70,6 \%$, en 1993); mientras que los de cuarto y más ordenes sólo alcanzan a $11,9 \%$.

Mortalidad infantil. Los fallecidos disminuyeron una vez más, por sexto año consecutivo desde 1988, para alcanzar un mínimo de 3438 y la tasa más baja registrada en la historia documentada del siglo: 11,9 por mil nacidos vivos (tabla 2). Según grupos de causas de muerte (tabla 3), todos los grupos se redujeron en forma

\section{Hospital San Juan de Dios.}

sustancial en los 25 años presentados, con excepción de las anomalías congénitas y los traumatismos y violencias; sin embargo, la tasa de estos últimos disminuyó, después de haber aumentado. Información no presentada muestra que proporciones cercanas al $40 \%$ de las muertes respiratorias de menores de un año son extrahospitalarias y eventualmente reducibles.

Mortalidad neonatal. Pese a que las muertes no alcanzan a dos mil y fueron ochocientas menos que en 1988, la tasa se redujo en forma mínima, permaneciendo prácticamente estacionaria (tabla 4).

Mortalidad infantil tardía. La tasa disminuyó en un punto, llegando al valor más bajo registrado y las muertes se bajaron en más de trescicntas (tabla 5).

Tasas en el país. Las tasas nacionales están muy influidas por las de la Región Metropolitana, variables entre sí y que concentran la mayoría de los nacimientos y muertes (tabla 6). Al igual que en años anteriores, se mantuvo gran dispersión, con los resultados más favorables en el Servicio Oriente de Santiago y los más desfavorables en Aysen. El extremo norte aparece en una situación favorable en relación al país, ventaja que se pierde en Antofagasta y Atacama. Arica muestra lo ya observado en otras situaciones, esto es que la mortalidad infantil es capaz-probablemente dentro de ciertos límitesde superar situaciones de alguna adversidad, pues mientras en los últimos años la economía 
Tabla 1

Natalidad, Chile 1980-1994

\begin{tabular}{lcc}
\hline Años & Nacidos vivos corregidos & Tasa natalidad* \\
\hline 1980 & 253581 & 22,3 \\
1981 & 260273 & 23,0 \\
1932 & 274600 & 23,8 \\
1983 & 260655 & 22,2 \\
1984 & 265016 & 22,2 \\
1985 & 261978 & 21,6 \\
1986 & 272997 & 22,1 \\
1987 & 279762 & 22,3 \\
1988 & 296581 & 23,3 \\
1989 & 3113798 & 23,4 \\
1990 & 307522 & 23,3 \\
1991 & 299456 & 22,4 \\
1992 & 293787 & 21,6 \\
1993 & 290438 & 21,0 \\
1994 & 288123 & 20.5 \\
.20 &
\end{tabular}

* Tasa por 1000 habitantes

Tabla 2

Mortalidad infantil, Chile 1980-1994

\begin{tabular}{lcc}
$\begin{array}{c}\text { Años } \\
\text { Defunciones } \\
\text { menores de 1 año }\end{array}$ & $\begin{array}{c}\text { Tasa } \\
\text { mortalidad infanti] }\end{array}$ \\
\hline 1980 & 8072 & 31,8 \\
1981 & 7082 & 27,2 \\
1982 & 6487 & 23,6 \\
1983 & 5705 & 21,9 \\
1984 & 5182 & 19,6 \\
1985 & 5105 & 19,5 \\
1986 & 5220 & 19,1 \\
1987 & 5182 & 18,5 \\
1988 & 5598 & 18,9 \\
1989 & 5183 & 17,1 \\
1990 & 4915 & 16,0 \\
1991 & 4385 & 14,6 \\
1992 & 4209 & 14,3 \\
1993 & 3792 & 13,1 \\
1994 & 3438 & 11,9 \\
\hline
\end{tabular}

* Tasa por $1000 \mathrm{NV}$ corregidos

de la región estuvo afectada por problemas que generaron una fuerte reacción local, la tasa de mortalidad infantil fue una de las dos más bajas de Chile. Coquimbo y San Felipe-Los Andes se sitúan algo mejor que el conjunto nacional, mientras que la $\mathrm{V}$ region lo hace peor.

Los servicios comprendidos entre la región del Libertador O'Higgins y Valdivia, con excepción de Talcahuano, se mantienen sobre las ta- sas, nacionales y conforman una macrorregión de alta población y situación más desfavorable. Lo inverso - tasas más bajas- ocurre con la región comprendida entre Llanquihue y Punta Arenas, excepto Aysen, que presenta las peores tasas del país. La situación de Magallanes es bastante particular, pues conjuga una bajísima tasa de mortalidad infantil tardía con una muy alta de mortalidad neonatal y en los últimos años ha presentado inestabilidad en sus posiciones dentro del concierto nacional ${ }^{2}$. Será interesante observar los datos de 1995, año en que la región se vio asolada por una catástrofe invernal natural (el llamado "terremoto blanco") que podría deteriorar fuertemente la economía de la zona.

\section{Comentario}

En la medida que se obtienen tasas por unjdades geográfico-administrativas de menor tamaño, la información para la gestión se va haciendo más exacta y correlacionada con la realidad que se pretende mejorar. Sin embargo, cuando se desagrega por unidades de tamaño muy pequeño o con población muy escasa, se produce cierta inestabilidad de las tasas, dado que pequeñas variaciones de los números pueden generar grandes oscilaciones de ellas. Es posible que ello explique parte de la volatilidad de las tasas de Magallanes.

En la perspectiva nacional se mantiene la desigualdad, pues en Aysen los resultados son radicalmente diferentes que en los servicios de salud Metropolitano Oriente y de Arica. Al decidir la inversión en salud existe la tendencia na-

Tabla 3

Mortalidad infantil según grupos de causas - Chile 1970-1994

\begin{tabular}{lrrrr}
\hline Grupos de causas & 1970 & 1980 & 1990 & 1994 \\
\hline Afecciones perinatajes & 16,77 & 12,54 & 5,53 & 4,16 \\
Anomalias congenitas & 3,25 & 3,86 & 3,66 & 3,35 \\
A. respiratorio & 27,94 & 5,02 & 2,59 & 1,42 \\
Traum. y envenenamientos & 1,13 & 2,03 & 2,36 & 1,57 \\
E. infecciosas y paras. & 20,06 & 3,47 & 0,60 & 0,38 \\
Resto & 10,17 & 4,91 & 1,24 & 1,05 \\
\hline Total & 79,30 & 31,80 & 16,00 & 11,9
\end{tabular}

*Tasas por l $000 \mathrm{NV}$ 
Tabla 4

Mortalidad neonatal - 1980-1994

\begin{tabular}{ccc}
\hline Años & $\begin{array}{c}\text { Defunciones } \\
\text { menores 28 dias }\end{array}$ & $\begin{array}{c}\text { Tasa mortalidad } \\
\text { neonatal }\end{array}$ \\
\hline 1980 & 4146 & 16,3 \\
1981 & 3458 & 13,3 \\
1982 & 3,377 & 12,3 \\
1983 & 2799 & 10,3 \\
1984 & 2449 & 9,2 \\
1985 & 2582 & 9.9 \\
1986 & 2655 & 9,7 \\
1987 & 2691 & 9,6 \\
1988 & 2814 & 9.5 \\
1989 & 2767 & 9,1 \\
1990 & 2607 & 8,5 \\
1991 & 2360 & 7,9 \\
1992 & 2254 & 7,7 \\
1993 & 2007 & 6,9 \\
1994 & 1973 & 6,8 \\
\hline
\end{tabular}

* Tasas por l 000 NV corregidos.

\section{Tabla 5}

Mortalidad infantil tardía, Chile 1980-1994

\begin{tabular}{lcc}
\hline Años & $\begin{array}{c}\text { Defunciones } \\
\text { 28 días a 11 meses }\end{array}$ & $\begin{array}{c}\text { Tasa mortalidad } \\
\text { infantil tardía* }\end{array}$ \\
\hline 1980 & 3926 & 15,5 \\
1981 & 3624 & 13,9 \\
1982 & 3110 & 11,3 \\
1983 & 2906 & 11,1 \\
1984 & 2733 & 10,3 \\
1985 & 2523 & 9,6 \\
1986 & 2565 & 9,4 \\
1987 & 2491 & 8,9 \\
1988 & 2784 & 9,4 \\
1989 & 2416 & 8,0 \\
1990 & 2307 & 7,5 \\
1991 & 2025 & 6,8 \\
1992 & 1955 & 6,6 \\
1993 & 1785 & 6,1 \\
1994 & 1465 & 5,1
\end{tabular}

* Tasas por $1000 \mathrm{NV}$ corregidos.

tural a hacerlo donde se obtenga mejor impacto para el país, criterio que -de ser seguido con rigurosidad-inclinarła siempre la inversión hacia áreas más pobladas y mantendría en desmedro a la undécima region. Como es natural, se necesita balancear el criterio de rendimiento de la inversión con el de equidad, dando mấs donde son mayores las necesidades.
El país tiene tasas de mortalidad satisfactorias para su grado de desarrollo, pero aún muy susceptibles de mejoría. Junto a los éxitos en la erradicación del sarampión y la poliomielitis, se mantienen vigentes los problemas respiratorios, y los traumatismos y violencias deben ser vigilados cuidadosamente para acentuar la tendencia a la reducción.

Tal vez no esté de más recordar que el descenso de la mortalidad infantil se traduce tambien en un incremento de los niños con enfermedades crónicas -estimados en más de 500000 por el Comité de Crónicos de la Sociedad de Pediatría- y que la reducción de las muertes no significa necesariamente una reducción de la morbilidad ni una mejoría de la calidad de vida.

\section{Tabla 6}

Mortalidad infantil según Servicio de Salud, Chile, 1994

Tasas por $\mathrm{t} 000$ nacidos vivos

\begin{tabular}{|c|c|c|c|}
\hline \multirow[b]{2}{*}{ Servicio } & \multicolumn{3}{|c|}{ Mortalidad } \\
\hline & Infantil & $\begin{array}{c}\text { Infantil } \\
\text { tardía }\end{array}$ & Neonatal \\
\hline Arica & 8,0 & 3,5 & 4,5 \\
\hline Iquique & 9.3 & 4.3 & 5,0 \\
\hline Antofagasta & 13.5 & 5,6 & 7.9 \\
\hline Alacama & 14,1 & 6,4 & 7.7 \\
\hline Coquimbo & 11,7 & 4,7 & 7,0 \\
\hline San Felipe-Los Andes & 10.9 & 4,1 & 6.8 \\
\hline Valparafso-San Antonio & 13,0 & 6,3 & 6.7 \\
\hline Vif́a-Quillota & 12.7 & 4,7 & 8,0 \\
\hline \multicolumn{4}{|l|}{ R, Metropolitana: } \\
\hline Norte & 11,2 & 5,3 & 5,9 \\
\hline Occidente & 11,2 & 4,7 & 6,4 \\
\hline Central & 8,8 & $3, \mathrm{~B}$ & 5,0 \\
\hline Oriente & 7,5 & 3,1 & 4,4 \\
\hline Sur & 10,7 & 4,4 & 6.3 \\
\hline Sur Orienle & 8,9 & 4,9 & 4,8 \\
\hline Lib. B. O'Higgins & 12,5 & 4,6 & 7,9 \\
\hline Maule & 13,1 & 5.8 & 7.2 \\
\hline Nuble & 13,1 & 6,1 & 7,0 \\
\hline Concepcióo-Arauco & 13,8 & 6,1 & 7.7 \\
\hline Talcatuano & 10,1 & 4,1 & 6.0 \\
\hline Bío-Bío & 13.0 & 5,4 & 7,6 \\
\hline Araucanía & 14,9 & 6.8 & 8,1 \\
\hline Valdivia & 12,7 & 5,0 & 7,7 \\
\hline Osorno & 10,6 & 4,8 & 5,8 \\
\hline Llanquihue-Chiloé & 10,7 & 4,5 & 6,2 \\
\hline Aysen & 17.6 & 7,5 & 10,0 \\
\hline Magallanes & 11.6 & 1,5 & 10,1 \\
\hline
\end{tabular}

* Tasas por $1000 \mathrm{NV}$. 


\section{Resumen}

Sc presentan las tasas de mortalidad infantil (11.9 por mil nacidos vivos), neonatal $(6,8$ por mil) e infantil tardía (5,1 por mil) de Chile, de 1994, a nivel nacional y desagregadas para los veintiséis servicios de salud estatales del país. Todas las tasas alcanzaron el valor más bajo observado desde que existe registro uniforme y confiable, pero mantienen una variación importante entre las diferentes regiones $y$ áreas del país; de esta manera de configuran macrorregiones de cierta homogeneidad en sus tasas, distintas a los valores del pais. Para una descripción mejor se presenta la serie histórica de tasas nacionales desde 1980 a 1994.

(Palabras clave: mortalidad infantil, mortalidad neonatal, Chile.)

\section{Agradecimientos}

El Programa Infantil del Ministerio de Salod autorizó el uso de este material para su difusion en la comunidad pediátrica.

\section{Referencias}

1. Anónimo: Programa Infantil. Diagnóslico de Situación. Documento del Ministerio de Salud de Chile 1995.

2. Vargas NA, Cerda J: Mortalidad infantil y neonatal en Chile entre 1965 y 1990. Rev Chil Petiatr 1993; 64: 45-49.

Esta publicación está disponible en copias de microfilms de 16 y 35 $\mathrm{mm}$ y nucrofichas de $105 \mathrm{~mm}$, las que pueden solicitarse a:

University Microfilms International

300 North Zeeb Road

Ann Arbor, Michigan 48106, USA.

This journal is also available in $16 \mathrm{~mm}$ microfilm, $35 \mathrm{~mm}$ microfilm and 105 mm microfilm copies through

University Microfilms International,

300 North Zeeb Road,

Ann Arbor, Michigan 48106, USA. 